\title{
The additive prognostic value of perfusion and functional data assessed by quantitative gated SPECT in women
}

\author{
Yves G. C. J. America, MD, ${ }^{a}$ Jeroen J. Bax, $M D$, PhD, ${ }^{a}$ Eric Boersma, $M S c, P h D,{ }^{b}$ \\ Marcel Stokkel, $M D, P h D,{ }^{c}$ and Ernst E. van der Wall, $M D, P^{a}$
}

Background. The aim of this study was to assess the prognostic value of technetium-99m tetrofosmin gated SPECT imaging in women using quantitative gated single photon emission computed tomography (SPECT) imaging.

Methods. We followed 453 consecutive female patients. Average follow-up was 1.33 years (max. 2.55). Hard endpoints were cardiac death, acute myocardial infarction, or documented ventricular fibrillation. Event-free survival curves were obtained. Optimal cutoff values for left ventricular $(L V)$ volumes, $L V$ ejection fraction $(L V E F)$, and perfusion data to predict outcome were determined by ROC curve analysis.

Results. A total of 236 patients had an abnormal study, of whom 27 patients experienced hard events (16 deaths) and 47 patients soft events. For hard events summed stress score (SSS) and LVEF, and for any cardiac event SSS showed independent incremental prognostic value. The survival curves were maximally separated when using cutoff values for SSS of $\geq 22$ and LVEF $<52 \%(P<0.001$, HR 4.61 and $P<0.001$ HR 5.24 for SSS and LVEF resp.), and SSS $\geq 14(P<0.001$ HR 3.76) for any cardiac event.

Conclusion. In women, perfusion and functional parameters derived from quantitative gated technetium-99m tetrofosmin SPECT imaging can adequately be used for cardiac risk assessment. Using quantitative gated SPECT, female patients with an LVEF $<52 \%$ or an SSS $\geq 22$ are at increased risk for subsequent hard events. Furthermore, patients with an SSS $\geq 14$ are at increased risk for any cardiac events. (J Nucl Cardiol 2009;16:10-9)

Key Words: Women $\cdot$ gated single photon emission computed tomography $\cdot$ prognosis

\section{INTRODUCTION}

Coronary artery disease (CAD) is the major cause of morbidity and mortality in the western countries. Increased mortality and reinfarction have been noted in women after myocardial infarction compared to men. ${ }^{1-3}$ The large proportion of atypical symptoms, higher incidence of associated disease (e.g., hypertension,

From the Department of Cardiology, ${ }^{\text {a }}$ Department of Radiology, Division of Nuclear Medicine, ${ }^{\mathrm{c}}$ Leiden University Medical Center, Leiden, The Netherlands and Thorax Center Rotterdam, ${ }^{\mathrm{b}}$ Rotterdam, The Netherlands.

Received for publication Jan 21, 2008; final revision accepted Jul 31, 2008.

Reprint requests: Ernst E. van der Wall, MD, PhD, Department of Cardiology, C5-P28, Leiden University Medical Center, Albinusdreef 2, 2300 RC Leiden, The Netherlands; e.e.van_der_wall@ lumc.nl.

$1071-3581 / \$ 34.00$

Copyright $₫ 2008$ The Author(s). This article is published with open access at Springerlink.com.

doi:10.1007/s12350-008-9012-6 diabetes mellitus), and the higher age at presentation may account for the worse outcome., ${ }^{3,4}$ Appropriate noninvasive diagnostic testing is important in the early diagnosis and the risk stratification of women with suspected CAD. Exercise ECG has a lower diagnostic and prognostic accuracy in women. It is influenced by multiple factors, i.e., exercise capacity and hormonal status. ${ }^{4,5}$ The increased age at presentation is often associated with lower exercise capacity and an inability to attain maximal stress. ${ }^{4,5}$ Myocardial perfusion imaging provides incremental prognostic information. ${ }^{4}$

The addition of regional left ventricular (LV) function parameters by gated single photon emission computed tomography (SPECT) improved the diagnostic accuracy and prognostic value of perfusion imaging, whereby LV function parameters have incremental prognostic value over perfusion data alone ${ }^{6-10}$ Previous studies set normal functional data limits. However, some problems still have to be solved. First, most data on prognostic value of the parameters assessed by gated 
SPECT have been obtained in a mixed gender population and may not be applicable to women. Women often have smaller LV volumes. It has been shown that gender related differences in normal limits exist. ${ }^{11,12}$ However, a multicenter phantom study showed a wide range of results in different standard end-systolic and end-diastolic volume combinations. Moreover, the LV ejection fraction (LVEF) was overestimated and both the endsystolic volume (ESV) and end-diastolic volume (EDV) were underestimated. Especially, this is the case for small volumes. Cutoff values for LV functional parameters should be validated in each center. ${ }^{13}$

Second, most data on prognostic value of perfusion analyses by gated SPECT is obtained by a 20 -segment model. However, recent guidelines on cardiac imaging suggest the use of a 17-segment model. Berman et al ${ }^{14}$ showed that a 17-segment model provides a more accurate prognostic categorization of individual patients with small abnormalities of the distal short axil and apical portions of the LV.

At present, few data exist on the prognostic value of gated SPECT in women. In this category of patients, little data are available on the value of LV function parameters to potentially improve risk stratification. Accordingly, the aim of the study was to assess the incremental prognostic value of technetium-99m tetrofosmin $\left(\mathrm{Tc}^{99 \mathrm{~m}}\right)$ gated SPECT imaging in women.

\section{METHODS}

\section{Study Population}

We studied 453 consecutive women (median age 62 years [53 to 70 years, 25 th to 75 th percentiles]) who underwent rest/ stress technetium-99m tetrofosmin myocardial perfusion gated SPECT imaging between October 1, 1999, and January 1, 2000, at the Leiden University Medical Center. Patients were followed up until the fixed census date May 1, 2000.

\section{Stress Myocardial Perfusion Protocol}

All patients were instructed to refrain from caffeinecontaining products for 24 hours before the test. Beta-blocking agents, nitrates, calcium antagonists were discontinued at least 48 hours prior to SPECT imaging. A symptom-limited bicycle exercise stress test was performed. Technetium-99m tetrofosmin (GE, Amersham, UK) was injected at peak stress, and exercise was continued for an additional 60 seconds. The exercise stress protocol includes a stepwise increase in workload depending on gender, age, weight, and height. This protocol is routinely applied in our institution. When the prespecified maximum workload (depending on gender, age, weight, and height) is achieved, the physical validity is considered to be at least $100 \%$. Exercise stress endpoints were severe angina, physical exhaustion, dyspnea, sustained tachy-arrhythmias, exertional hypotension, or ischemic ST-T segment-depression of at least $0.2 \mathrm{mV}$ and a duration of 80 milliseconds. Exercise was considered inadequate if the physical validity of the patient was less than $80 \%$ of the predicted validity (workload) in the absence of angina or an ischemic ST-depression. Patients who were unable to perform a physical stress test or patients with a left bundle branch block underwent a pharmacological stress test as described previously. ${ }^{15}$ Vasodilatation was induced using intravenous administration of adenosine at a dose rate of $0.14 \mathrm{mg} / \mathrm{kg} / \mathrm{min}$ for 6 minutes. Technetium- $99 \mathrm{~m}$ tetrofosmin was injected 3 minutes after start of infusion of the pharmacological agent. Dobutamine $0.3 \mathrm{mg} / \mathrm{kg}$ infusion during 15 minutes was used in patients who were not able to exercise adequately and in whom the use of adenosine was contraindicated (except patients with LBBB). Horizontal or downsloping ST-segment depression of $1 \mathrm{~mm}$ or greater or upsloping of $1.5 \mathrm{~mm}$ or greater at $80 \mathrm{mil}-$ liseconds after $\mathbf{J}$ point was considered positive for ischemia. In case of ST-T-segment abnormalities, registration of the stress ECG was continued until normalization of the ECG was seen.

\section{Gated SPECT Acquisition Protocol}

Both 1-day and 2-day imaging protocols were used. ${ }^{15} \mathrm{~A}$ dose of $500 \mathrm{MBq}$ technetium-99m tetrofosmin was given intravenously for the 2-day protocol stress study and $750 \mathrm{MBq}$ for the 1-day protocol stress study. Dosages of 500 and $250 \mathrm{MBq}$, respectively, were given for the rest study. All acquisitions took place 30 to 45 minutes (stress) or 45 to 60 minutes (rest) post-injection. Gated SPECT acquisition protocol was performed post-stress. In case of 1-day protocol the rest study was done first. Imaging was performed with a triple-head $360^{\circ}$ rotating gamma camera (Toshiba GC-9300 GMS, Japan). A total of 90 frames of 30' duration using a $64 \times 64$ pixel matrix were obtained at 4-degree intervals using a non-circular orbit. Sixteen bins per cardiac cycle were acquired. All studies were pre-filtered using a 9-order Butterworth filter at a cutoff frequency of 0.32 cycles/pixel (rest) [pixel size $6 \mathrm{~mm}$ ] or 0.26 cycles/pixel (stress). All images were subject to quality control measures, including cinematic display for assessment of patient motion, corrections for field non-uniformity, and center of rotation. No attenuation or scatter correction was used. The reconstructed data were projected as tomographic slices in short-axis and vertical/ horizontal long-axis views. Myocardial perfusion data and quantitative LV volumes and LVEF were calculated using the commercially available Cedars-Sinai's Quantitative gated SPECT (QGS) software, version 2.0, revision A', 7,8 When automatic reconstruction or reorientation failed, reconstruction limits and axes were assigned manually. End-systolic volume index (ESVi) and EDV index (EDVi), in milliliters per square meter, were derived by dividing ESV and EDV by body surface area (BSA), respectively.

\section{Semi-Quantitative Visual Analysis of Myocardial Perfusion SPECT}

Semi-quantitative visual interpretation of SPECT perfusion images used short-axis and vertical long-axis tomograms 
divided into 17 segments for each patient. ${ }^{14}$ These segments were assigned to four evenly spaced regions in apical, six midventricular, and six basal slices of the short-axis views and one apical segment on the mid-ventricular vertical long-axis slice. Each segment was scored using a five-point scoring system $(0=$ normal,$\quad 1=$ equivocal, $2=$ moderate,$\quad 3=$ severe reduction of radioisotope uptake, $4=$ absence of detectable radiotracer in a segment). Apparent perfusion defects presumably caused by soft tissue attenuation were assigned a score of 1 . The observers were blinded to the patient's clinical history and results of stress testing.

Three global perfusion indices were employed to combine assessments of defect extent and severity, ${ }^{16}$ namely summed stress score (SSS), summed rest score (SRS), and summed difference score (SDS). These indices were converted to a percentage of the total myocardium involved with ischemic, or fixed defects. ${ }^{17,18}$ All studies were evaluated by two experienced observers in consensus readings.

\section{Patient Follow-up and Endpoint Definitions}

Both medical records and the automated hospital information system were reviewed. If these data did not cover the entire period from recruitment until census date, the patient was sent a questionnaire. In case of no response, a second questionnaire was sent after 3 months. Cardiac death (CD) was noted as death due to any cardiovascular cause, confirmed by certificate and hospital chart of physician's records; in addition, hard events were defined as cardiac death, nonfatal myocardial infarction (MI), or documented ventricular fibrillation. An acute MI was documented by appropriate ECG findings, accompanied with serum cardiac enzyme level changes or isolated cardiac enzyme level changes. The cardiac enzymes used were creatine kinase $(\mathrm{CK})$ and troponin $\mathrm{T}$. Soft events were defined as any late ( $>60$ days after MPI) revascularization procedure, i.e., coronary artery bypass grafting (CABG) or percutaneous transluminal coronary angioplasty (PTCA).

\section{Statistical Analysis}

Most continuous variables had non-normal distribution (as evaluated by Kolmogorov-Smirnov tests). For reasons of uniformity, summary statistics for all continuous variables are therefore presented as medians together with the 25th and 75th percentiles. Categorical data are summarized as frequencies and percentages. Differences in baseline characteristics between patients who reached the primary endpoint and those who did not were analyzed using Wilcoxon-Mann-Whitney tests, Chi-square tests, or Fisher's exact tests, as appropriate.

Univariable and multivariable Cox proportional hazard regression analyses were applied to study the relation between perfusion and functional data (based on gated SPECT imaging) and the incidence of study endpoints over time. We considered a broad range of potential confounders of these relations, including age, smoking, family history of CAD, hypertension, diabetes, hyperlipidemia, established CAD, resting heart rate, type of stressor (pharmacological or exercise), level of exercise, presenting symptom (angina pectoris), presence of ischemia on the exercise ECG. Cox models aiming at prediction should be used with a minimum of 10 events per predictor variable (EPV). Simulation studies showed increasing bias and variability, unreliable confidence interval coverage, and problems with model convergence as EPV declined below 10. ${ }^{19,20}$ However, Vittinghoff and McCulloch demonstrated that this rule of thumb is too conservative in analyses of causal influences based on observational data, and control of confounding may require adjustment for more covariates than the rule of 10 EPV allows. ${ }^{21}$ Discounting of statistically significant associations from models that are based on 5 to $9 \mathrm{EPV}$ and that aim to adjust for confounding is not justified. Hence, multivariable models that are based on our dataset (with 27 events) could safely contain 3 to 5 variables. We performed stepwise regression according to the backward deletion approach, applying a $P$-value of 0.15 as threshold for variable removal, to adjust for as many covariables as is reasonably allowed by the (limited) number of events. We report crude and adjusted hazard ratios (HR) and corresponding 95\% confidence intervals (CI).

It was not our intention to formally build an outcome prediction model. Still, all the continuous variables were assessed for linearity by entering a transformed variable in addition to the variable of interest. The natural logarithm and square transformations were used. A significant change in the -2 log-likelihood was considered as a sign of non-linearity, otherwise the linearity assumption was accepted. All variables met the linearity assumption. To check the proportional hazard assumption, i.e., that the hazard ratio for two subjects with fixed predictors is constant over time, $\log (-\log$ [survival probability]) for different categories was plotted against time to ensure that the curves were reasonably parallel. In general, all proportionality assumptions were appropriate.

It appears that SSS and LVEF were associated with the incidence of study endpoints over time. We applied receiver operator characteristic (ROC) curve analyses to determine the optimal cutoff values for these variables that can be used for event prediction in clinical practice. Optimal cutoff values were defined as values resulting in the maximal sum of sensitivity and specificity. Subsequently, event-free survival curves were obtained according to the method of Kaplan and Meier, separating patients with a value below or above the threshold. Differences in event-free survival between patients were evaluated by log-rank tests.

For all analyses, a $P$ value $<0.05$ was considered statistically significant. No correction was made to adjust for multiple comparisons.

\section{RESULTS}

\section{Patient Characteristics}

Four-hundred and fifty-three patients were followed. The patient baseline characteristics are shown in Table 1. Median age was 62 (53 to 70 years [25th to 75th percentiles]). One-hundred and fourteen (26\%) patients had known CAD. Of those patients 67 (15\%) 
Table 1. Patient characteristics

\begin{tabular}{ll}
\hline $\begin{array}{l}\text { Total number of patients } \\
\text { Age (years)* }\end{array}$ & 453 \\
Risk factors for CAD & $62(53-70)$ \\
Family history CAD & $65 \%$ \\
Hyperlipidemia & $61 \%$ \\
Hypertension & $60 \%$ \\
Diabetes & $18 \%$ \\
Smoking & $43 \%$ \\
Body Mass Index ${ }^{*}$ & $26.9(26.1-28.8)$ \\
Body Mass Area $\left(\mathrm{m}^{2}\right)^{*}$ & $1.8(1.7-2.5)$ \\
History CAD & $114(26 \%)$ \\
Myocardial infarction & $67(15 \%)$ \\
Revascularization & $67(15 \%)$ \\
\hline
\end{tabular}

${ }^{*}$ Median (25th-75th percentiles).

$C A B G$, Coronary artery bypass graft surgery; $C A D$, coronary artery disease.

Body Mass Index according to the Mosteller Formula.

had sustained a MI, 67 (15\%) experienced one or more revascularization procedures, and none had a history of cardiac arrest.

\section{Perfusion and Function}

Perfusion and function data are shown in Table 2. Two-hundred and thirty-five patients $(48 \%)$ had an abnormal perfusion study. Of those, 105 patients (45\%) showed an ischemic perfusion study. The median SSS was 5 (25th to 75 th percentiles: 0 to 22), the median SRS was 3 (25th to 75 th percentiles: 0 to 17 ), and the median SDS was 2 (25th to 75 th percentiles: 0 to 3 ); median

Table 2. Findings at gated SPECT imaging

\begin{tabular}{lccc}
\hline & Median & $\begin{array}{c}\text { 25th-75th } \\
\text { percentile }\end{array}$ & Range \\
\hline \multicolumn{3}{l}{$\begin{array}{l}\text { Total patient } \\
\text { Myoup (n=453) }\end{array}$} \\
\multicolumn{2}{l}{$\begin{array}{l}\text { Myocardial perfusion } \\
\text { SSS }\end{array} \quad 5$} & $0-22$ & $0-67$ \\
SRS & 3 & $0-17$ & $0-65$ \\
SDS & 2 & $0-3$ & $0-34$ \\
Left ventricular function & & \\
EDV (ml) & 70 & $56-89.5$ & $32-343$ \\
ESV (ml) & 24 & $16-39.5$ & $4-298$ \\
LVEF (\%) & 65.5 & $54-73$ & $13-91$ \\
ESVi & 13 & $9-22$ & $2.0-156$ \\
EDVi & 38 & $32-49$ & $18-180$
\end{tabular}

SSS, Summed stress score; SRS, summed rest score; SDS, summed difference score; $E D V$, end-diastolic volume; $E S V$, end-systolic volume; $L V E F$, left ventricular ejection fraction; $E S V i$, end-systolic volume index; EDVi, end-diastolic volume index.
EDV was $70 \mathrm{~mL}$ (25th to 75 th percentiles: 56 to $89.5 \mathrm{~mL}$ ), median ESV $24 \mathrm{~mL}$ (25th to 75 th percentiles: 16 to $39.5 \mathrm{~mL}$ ), and median LVEF $65.5 \%$ (25th to 75 th percentiles: $54 \%$ to $73 \%$ ). The median EDVi was 38 (25th to 75th percentiles: 32 to 49) and the median ESVi 13 (25th to 75 th percentiles: 9 to 22 ).

\section{Outcome}

In 422 patients $(93.2 \%)$ follow-up was complete until census or death. The average follow-up was 1.33 years (maximum 2.55 years). Twenty-seven patients suffered a hard event during follow-up, on average after 1.06 years (range 1 day to 2.50 years). Sixteen patients died of cardiac cause during follow-up, with a mean follow-up of 0.72 years (range 1 day to 2.22 years). No hard events occurred during surgery or coronary intervention. Forty-seven soft events were recorded (mean follow-up 1.43 years; range 93 days to 2.52 years): 15 patients underwent coronary bypass surgery, and in 32 patients PTCA was performed.

Patients with hard events during follow-up had significant larger LV volumes, larger perfusion defects and lower LVEF than those without hard events. Patients with any cardiac event during follow-up had significantly larger LV volumes, more extensive perfusion defects, lower LVEFs, and larger reversible defects than those without (Table 3).

Results based on univariate analysis are listed in Table 4. Summed stress score, SRS, all the function variables (EDV, ESV, EDVi, ESVi, and LVEF) were predictive for $\mathrm{CD}$ and hard events. For hard events diabetes, smoking, presenting symptom (angina pectoris), and pharmacological stress test were also predictive. Age, diabetes, hyperlipedemia, smoking, history of MI, presenting symptom, pharmacological stress test, level of exercise, and resting heart rate were predictive for any cardiac event. Also, larger LV volumes (EDV and ESV), decreased LVEF, larger perfusion defects, and ischemia were predictive for any cardiac event.

The variables entered in the initial multivariable model for hard events included age, diabetes, hypertension, family history, hyperlipedemia, smoking, presenting symptom, history of CAD, history of MI, history of revascularization, pharmacological stress test, level of exercise, SSS, SRS, and all the function variables (EDV, ESV, EDVi, ESVi, and LVEF). For any cardiac events age, diabetes, hypertension, family history, hyperlipedemia, smoking, presenting symptom, known CAD, history of MI, history of revascularization, pharmacological stress test, resting heart rate, level of exercise, ischemia on stress ECG, SSS, SRS, and all the function variables (EDV, ESV, EDVi, ESVi, and LVEF) were entered in the model. 
Table 3. Outcome: perfusion and functional data

\begin{tabular}{|c|c|c|c|}
\hline & $\begin{array}{c}\text { Hard } \\
\text { events* }\end{array}$ & $\begin{array}{l}\text { No hard } \\
\text { event* }\end{array}$ & P-value \\
\hline \multicolumn{4}{|c|}{ Myocardial perfusion } \\
\hline SSS & $25(0-46)$ & $4(0-21)$ & $<0.01$ \\
\hline SRS & $18(2-30)$ & $2(0-15)$ & $<0.01$ \\
\hline \multicolumn{4}{|c|}{ Myocardial function } \\
\hline EDV & $85(70-164)$ & $69(56-87)$ & $<0.01$ \\
\hline ESV & $45(23-91)$ & $23(16-37)$ & $<0.01$ \\
\hline LVEF & $50(31-64)$ & $66(55-73)$ & $<0.01$ \\
\hline ESVi & $24(12-49)$ & $12(8-21)$ & $<0.01$ \\
\hline \multirow[t]{2}{*}{ EDVi } & $50(37-84)$ & $38(32-47)$ & $<0.01$ \\
\hline & $\begin{array}{l}\text { Any cardiac } \\
\text { events* }\end{array}$ & $\begin{array}{l}\text { No cardiac } \\
\text { event* }\end{array}$ & P-value \\
\hline \multicolumn{4}{|c|}{ Myocardial perfusion } \\
\hline SSS & $23(4-35)$ & $3(0-19)$ & $<0.01$ \\
\hline SRS & $12(2-$ & 14) & $<0.01$ \\
\hline SDS & $3(0-13)$ & $0(0-1)$ & $<0.01$ \\
\hline \multicolumn{4}{|c|}{ Myocardial function } \\
\hline EDV & 77 (66-103) & $68(56-86)$ & 0.01 \\
\hline ESV & $34(21-58)$ & $22(15-37)$ & $<0.01$ \\
\hline ESVi & $17(10-33)$ & $12(8-21)$ & $<0.01$ \\
\hline EDVi & $42(34-59)$ & $38(32-47)$ & 0.01 \\
\hline LVEF & $56(44-68)$ & $66(56-73)$ & $<0.01$ \\
\hline
\end{tabular}

*Values of the median (25th to 75th percentile).

Only parameters that showed significantly differences between the patient groups are mentioned.

SSS, Summed stress score; SRS, summed rest score; SDS, summed difference score; $E D V$, end-diastolic volume; $E S V$, end-systolic volume; $L V E F$, left ventricular ejection fraction; $E S V i$, end-systolic volume index; $E D V i$, end-diastolic volume index.

Multivariate analysis showed that summed stress score and LVEF provided independent and incremental prognostic value for hard events (beyond clinical data and/or perfusion variables, respectively). Summed stress score provided independent and incremental prognostic value for any cardiac event (Table 4).

\section{Survival}

The annual CD rate as function of percent stress defect for $<5,10$ to 20 , and $>20 \%$ hypoperfused myocardium was $<0.7,1.6$, and $4.6 \% / y e a r s$, respectively. The annual hard event rate for these subgroups are 1.0, 3.1 , and $8.3 \%$ year, respectively. ROC curve analysis was used to determine the optimal cutoff values for LVEF and SSS to predict outcome. For hard events optimal cutoff values for these different parameters could be determined. According to these cutoff values,
Table 4. Univariate and multivariate analysis of clinical data, perfusion, and functional data assessed by gated SPECT

\begin{tabular}{ccc}
$\begin{array}{c}\text { Hazard } \\
\text { ratio }\end{array}$ & $95 \% \mathrm{CI}$ & $\boldsymbol{P}$-value \\
\hline
\end{tabular}

Univariate analysis

Total mortality

$\begin{array}{llll}\text { Age } & 1.02 & 0.97-1.06 & \text { NS } \\ \text { Diabetes } & 2.58 & 0.75-8.80 & \text { NS } \\ \text { Hypertension } & 2.08 & 0.56-7.69 & \text { NS } \\ \text { Family history } & 0.79 & 0.22-2.79 & \text { NS } \\ \text { Lipid } & 0.39 & 0.12-1.22 & \text { NS } \\ \text { Smoke } & 1.23 & 0.55-2.75 & \text { NS } \\ \text { CAD } & 0.95 & 0.31-2.93 & \text { NS } \\ \text { Symptom (AP) } & 0.99 & 0.79-1.24 & \text { NS } \\ \text { History MI } & 1.93 & 0.62-6.00 & \text { NS } \\ \text { History revasc } & 0.00 & 0.00-0.00 & \text { NS } \\ \text { Pharmacological } & 1.37 & 0.97-1.93 & \text { NS } \\ \text { Resting HR } & 1.03 & 0.92-1.42 & \text { NS } \\ \text { Level of } & 0.90 & 0.85-1.00 & \text { NS } \\ \quad \text { exercise } & & & \\ \text { ECG ischemia } & 0.72 & 0.04-1.89 & \text { NS } \\ \text { SSS } & 1.04 & 1.01-1.07 & 0.01 \\ \text { SRS } & 1.04 & 1.01-1.07 & 0.01 \\ \text { SDS } & 1.05 & 0.98-1.13 & \text { NS } \\ \text { EDV (mL) } & 1.01 & 1.00-1.07 & <0.001 \\ \text { ESV (mL) } & 1.01 & 1.01-1.02 & <0.001 \\ \text { LVEF (\%) } & 0.95 & 0.92-0.97 & <0.001 \\ \text { EDVi } & 1.03 & 1.01-1.04 & <0.001 \\ \text { ESVi } & 1.03 & 1.02-1.04 & <0.001\end{array}$

Hard events

Age

1.02

0.98-1.05

NS

Diabetes

3.04

Hypertension

0.91

1.24-7.44

$0.40-2.11$

0.02

Family history

0.70

$0.28-1.72$

NS

Lipid

0.76

Smoke

1.80

CAD

1.51

$0.32-1.80$

NS

1.02-3.15

NS

0.67-3.40

0.04

Symptom (AP)

1.14

History MI

1.02-1.27

NS

2.14

History revasc

0.90-5.08

0.02

0.94

Pharmacological

2.53

Level of

0.96

$0.51-1.73$

NS

1.10-5.80

NS

0.91-1.00

0.03

exercise

Resting HR

0.99

ECG ischemia

1.32

0.96-1.02

0.54-3.24

SSS

1.05

SRS

1.05

SDS

EDV $(\mathrm{mL})$

1.05

1.01

1.03-1.07

1.03-1.08

0.99-1.11

1.01-1.02

1.01-1.02
NS

NS

NS

$<0.001$

$<0.001$

NS

$<0.001$ 
Table 4 continued

\begin{tabular}{lccl}
\hline & $\begin{array}{c}\text { Hazard } \\
\text { ratio }\end{array}$ & 95\% CI & P-value \\
\hline LVEF (\%) & 0.95 & $0.93-0.97$ & $<0.001$ \\
EDVi & 1.02 & $1.01-1.03$ & $<0.001$ \\
ESVi & 1.02 & $1.01-1.03$ & $<0.001$ \\
Any cardiac events & & & \\
Age & 1.03 & $1.01-1.05$ & 0.01 \\
Diabetes & 1.88 & $1.07-3.30$ & 0.03 \\
Hypertension & 1.00 & $0.59-1.67$ & NS \\
Family history & 1.21 & $0.69-2.11$ & $\mathrm{NS}$ \\
Lipid & 2.03 & $1.13-3.64$ & 0.02 \\
Smoke & 1.76 & $1.27-2.45$ & 0.001 \\
CAD & 2.27 & $1.37-3.75$ & 0.001 \\
Symptom (AP) & 1.11 & $1.04-1.20$ & 0.004 \\
History MI & 2.36 & $1.35-4.12$ & 0.003 \\
History revasc & 1.09 & $0.80-1.50$ & $\mathrm{NS}$ \\
Pharmacological & 1.82 & $1.10-3.00$ & 0.02 \\
Resting HR & 0.98 & $0.96-1.00$ & 0.03 \\
Level of & 0.97 & $0.94-0.99$ & 0.01 \\
exercise & & & \\
ECG ischemia & 1.42 & $0.81-2.48$ & $\mathrm{NS}$ \\
SSS & 1.05 & $1.03-1.06$ & $<0.001$ \\
SRS & 1.03 & $1.02-1.05$ & $<0.001$ \\
SDS & 1.11 & $1.08-1.14$ & $<0.001$ \\
EDV (mL) & 1.01 & $1.00-1.01$ & 0.01 \\
ESV (mL) & 1.01 & $1.00-1.01$ & 0.01 \\
LVEF (\%) & 0.98 & $0.96-0.99$ & $<0.001$ \\
EDVi & 1.01 & $1.00-1.02$ & 0.02 \\
ESVi & 1.01 & $1.00-1.02$ & 0.01 \\
Multivariate analysis & & & \\
Hard events & & & \\
LVEF (\%) & 0.97 & $0.94-1.00$ & 0.03 \\
SSS & 1.03 & $1.01-1.06$ & 0.02 \\
Any cardiac events & & & \\
SSS & 1.06 & $1.03-1.08$ & $<0.001$ \\
\hline Hardeventsmy & & & \\
\hline
\end{tabular}

Hard events: myocardial infarction, cardiac arrest, ventricular fibrillation and total mortality; Any cardiac event: myocardial infarction, cardiac arrest, ventricular fibrillation and total mortality, revascularization procedure; Symptom (AP): angina pectoris as presenting symptom; Pharmacological: pharmacological stress test; Resting HR: resting heart rate; Level of exercise: \% of the target level of exercise adjusted for gender, age, length and weight; ECG ischemia: ischemia on stress ECG.

$E D V$, End-diastolic volume; $E S V$, end-systolic volume; $E S V i$, end-systolic volume index; $E D V i$, end-diastolic volume index; $L V E F$, left ventricular ejection fraction; SSS, Summed stress score; SRS, summed rest score; SDS, summed difference score; $C A D$, coronary artery disease; $C I$, confidence interval; $M I$, myocardial infarction.

patients could be divided into two groups (Figures 1 and 2). An LVEF $<52 \%$ (area under the curve [AUC] 0.75) was associated with a significantly higher risk of hard

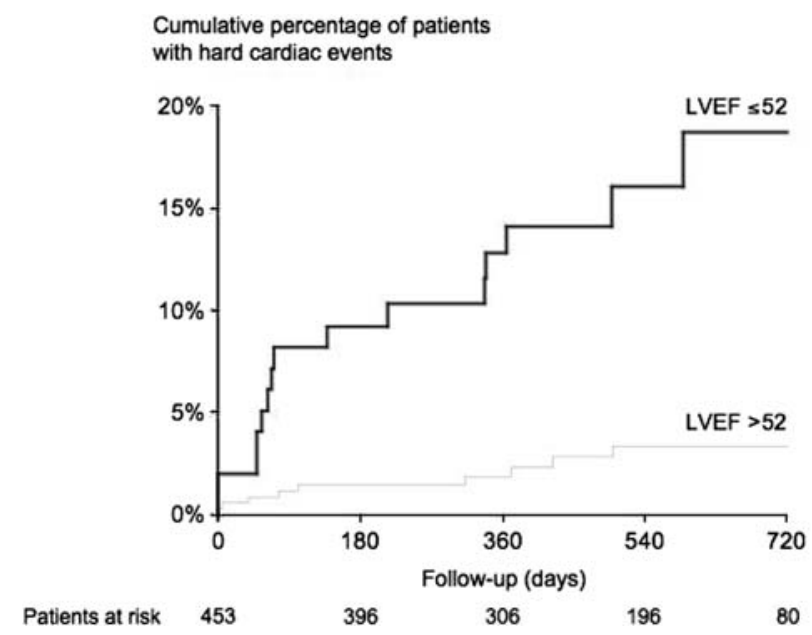

Figure 1. Hard endpoints according to the cutoff values for LVEF.

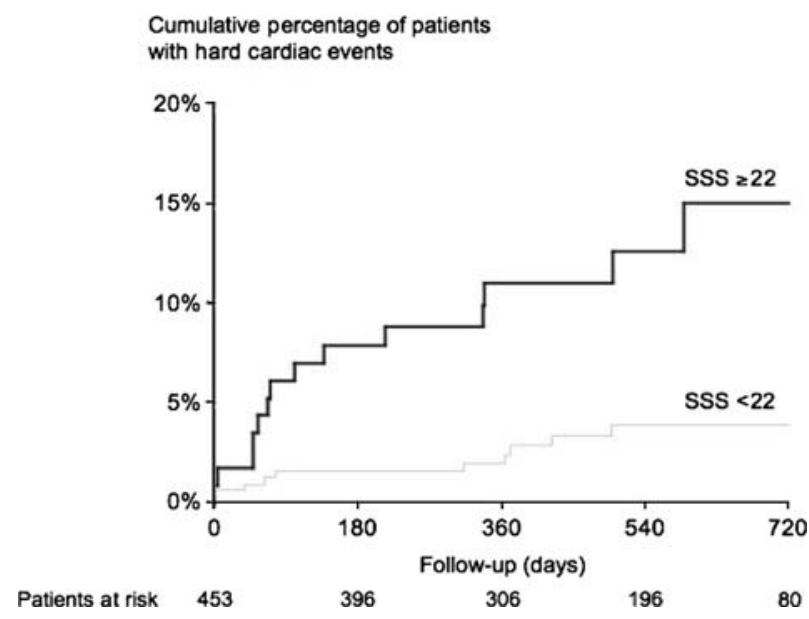

Figure 2. Hard endpoints according to the cutoff values for SSS.

events $(P<0.0001)$. Patients with an LVEF $<52 \%$ had an annual event rate of $11.6 \%$ with an annual $\mathrm{CD}$ rate of $6.6 \%$, whereas those with an LVEF $\geq 52 \%$ had an annual hard event rate of $1.7 \%$, and an annual CD rate of $0.6 \%$. For SSS, maximal separation could be found using a cutoff score of 22 (AUC $0.84 ; P=0.0001$ ). The annual hard event rate for patients with an SSS $\geq 22$ was $10.5 \%$, whereas those with an SSS $<22$ had an annual hard event rate of $2.5 \%$.

For any cardiac event optimal cutoff value for SSS could be determined (Figure 3). Maximal separation could be found using a cutoff score of 14 (AUC 0.81). The annual any cardiac event rate for patients with an SSS $\geq 14$ was $20.8 \%$, whereas those with an SSS $<14$ had an annual any cardiac event rate of $5.9 \%$ $(P<0.0001)$. 


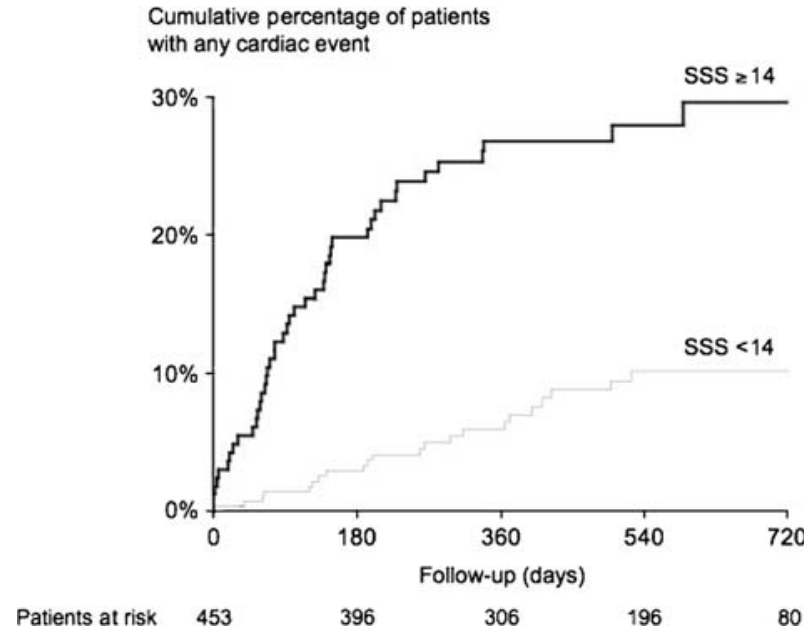

Figure 3. Any cardiac endpoint according to the cutoff values for SSS.

\section{DISCUSSION}

Our results show that relatively young female patients with hard events had significantly larger LV volumes, increased defect sizes, and lower LVEFs than those without hard events. Also, patients with any cardiac event during follow-up had significantly larger LV volumes, increased defect sizes, and lower LVEFs than those without any cardiac events. Moreover, patients with any cardiac event had significantly larger ischemic defects. For hard events and cardiac death SSS, SRS, and all the function variables (EDV, ESV, EDVi, ESVi, and LVEF) were predictive. For hard events diabetes, smoking, presenting symptom, and pharmacological stress test were also predictive. Age, diabetes, hyperlipedemia, smoking, history of MI, presenting symptom, resting heart rate, and pharmacological stress test were predictive for any cardiac event. Also, larger LV volumes (EDV and ESV), decreased LVEF, larger perfusion defects, and ischemia were predictive for any cardiac event. Summed stress score obtained by quantitative gated SPECT imaging had independent and incremental prognostic value for both hard events and any cardiac event in female patients beyond clinical data. For hard events also LVEF had independent and incremental prognostic value beyond clinical and perfusion data. Using these perfusion and functional data, female patients with increased risk of having subsequent serious cardiac events could be identified. Using ROC curve analysis, cutoff values for LVEF of 52\% and SSS 22 for hard events and SSS of 14 for any cardiac event yielded the highest sensitivity/specificity (discriminative power) to predict increased cardiac risk. An SSS $>22$ and an LVEF of $<52 \%$ were predictive for subsequent hard events. An SSS > 14 was predictive for any cardiac event.

To our knowledge, this is the first study to show that large ischemic and mixed defects are not all at high risk of events. Only a combination of perfusion and function allows to effectively risk stratify this population and give an individualized risk estimates for this heterogeneous population. Therefore, perfusion data per se are definitely not enough to make individualized prognostic statements.

\section{Prognostic Value of Perfusion Data}

Our perfusion data extend previous findings that average defect size was largest in those patients who suffered a hard event. In particular the SSS has been shown to be a powerful independent predictor of cardiac events. ${ }^{10,22-24}$

However, most of the observations have been made in a mixed gender population. ${ }^{10,22-24}$ Sharir et $\mathrm{al}^{11}$ showed that for CD and MI, perfusion data provided a larger proportion of the prognostic information for women than they did for men. We demonstrated that perfusion data have incremental value over clinical data, both for hard events and any cardiac event. In particular, women with moderate to severe perfusion defects had a high annual hard event rate. Those with a normal perfusion image have very low annual event rate. Stressinduced ischemia was predictive in women who suffered any cardiac event but had no incremental prognostic value for predicting hard events. This is in line with previous findings that suggest that the extent of defect size and functional abnormalities are more predictive for hard events (CD and MI), whereas the extent of ischemia is more predictive for soft events and/or MI. ${ }^{10,25-27}$ By using gated SPECT perfusion imaging, women at increased risk of having a cardiac event could be identified.

Most of the published data used a 20-segment approach. Berman et al ${ }^{14}$ showed that in a 17 -segment approach the apex and adjacent short-axis slice of the LV is less overweighed. There was a trend toward less frequent borderline scans with more normal and severely abnormal scans in the overall population. They found in the patients who shifted from abnormal scan by 20 segment model to normal scan in the 17-segment approach an equal cardiac death rate to other normal patients.

Berman et $\mathrm{al}^{14}$ found a significantly higher annual $\mathrm{CD}$ rate for women compared to men in group of patients with moderately to severe perfusion defects. We found the same annual $\mathrm{CD}$ rates for the patients with normal images (stress defect $<5 \%$ of the myocardium, $0.5 \%$ vs $<0.7 \%$ ). However, a smaller annual CD rate in 
the group of patients with severe perfusion defects (4.6\% vs $6.4 \%)$ was found. This may be explained by the fact that our population was 10 years younger. In a matched aged group, Groutars et $\mathrm{al}^{24}$ found similar event rates compared to our study ( $0.4 \%$ vs $0.7 \%$ annual $\mathrm{CD}$ rate for normal perfusion, $2.1 \%$ vs $2.4 \%$ annual CD for moderate to severely abnormal images). Similar results were found for hard cardiac events. Our optimal cutoff value to identify patients at high risk for hard events was 22 (32\% of the left ventricle). Patients with a SSS $<22$ had the same annual hard event rate demonstrated by Zellweger et $\mathrm{al}^{28}$ for mildly abnormal scans (2.5\% vs $2.4 \%)$. Travin et al ${ }^{10}$ reported similar annual hard event rates for patients with mildly abnormal perfusion images compared to patients with an SSS $<22$ in our study (2.5\% vs $3.0 \%)$. The cutoff value of SSS $>22$ suggests that the major part of the hard events occurs in patients with very large perfusion defects. Therefore, the group of female patients with severe perfusion defects is diverse with different outcomes. In our group, using our cutoff values, it is possible to identify females at increased risk.

A few data are reported on any cardiac events. Groutars et $\mathrm{al}^{29}$ reported good outcome in patients with normal perfusion studies. The annual event rate was $0.8 \%$ for any cardiac events. Schinkel et $\mathrm{al}^{30}$ found that for all cardiac events an abnormal scan and SSS had independent prognostic value, which is in line with our results.

\section{Prognostic Value of Functional Data}

Our data showed that functional data obtained from technetium-99m tetrofosmin gated SPECT were predictive for cardiac events.

We found that patients who suffered a cardiac event (for subgroups: cardiac death, hard events, and any cardiac event) during follow-up had significantly increased LV volumes and a significantly lower LVEF. Only for hard events functional data (LVEF) had independent incremental prognostic value. Our results underscore previously published data on the prognostic value of gated SPECT imaging. ${ }^{26-34}$

Sharir et $\mathrm{al}^{9}$ showed that perfusion variables and ESV were powerful markers in the prediction of total coronary events, whereas in the prediction of $\mathrm{CD}$, poststress LVEF and ESV were independent predictors and had incremental value over perfusion data. According to their criteria, patients were at increased cardiac risk when they had an LVEF $<45 \%$ and an ESV $>70 \mathrm{~mL}$. The study by Sharir et $\mathrm{al}^{9}$ was performed in a very large cohort of more than 1,600 patients, representing a heterogeneous population of patients with CAD in terms of LV function abnormalities.
Recently, Sharir et al ${ }^{11}$ showed lower limits of LVEF in women and men with a low likelihood of CAD-51\% (mean-2 SDs) and 43\%, respectively. They also report upper limits for ESVi and EDVi for both genders $\left(27\right.$ and $60 \mathrm{~mL} / \mathrm{m}^{2}$ for women and 39 and $75 \mathrm{~mL} / \mathrm{m}^{2}$ in men, respectively).

Compared to the data reported by Sharir et al, ${ }^{9}$ the annual event rate in our group of women with an LVEF $\geq 52 \%$ was similar to the annual event rate of a mixed population of patients with an LVEF $\geq 45 \%$ (regardless of the degree of perfusion abnormality).

To summarize, functional data obtained from technetium-99m tetrofosmin gated SPECT can be used to identify women at increased cardiac risk. The cutoff value for LVEF used in a mixed gender population is not applicable to studies in women. Women at increased risk of major adverse cardiac events, those with an LVEF $<52 \%$ and/or an SSS of $>22$, may benefit from more aggressive invasive therapy. However, further randomized prospective studies are needed to show better clinical outcome of aggressive therapy in these patients.

\section{Study Limitations}

It is known that in patients with small hearts, LV volumes, especially ESV, measured by the QGS technique, are often underestimated and LVEF is often overestimated. In general, this phenomenon occurs more in women than in men because women tend to have smaller LVs. However, previous studies ${ }^{11}$ showed a significant correlation between ESV and EDV vs BSA. Another study ${ }^{13}$ showed a systematic underestimation of volumes, more so for small volumes. As long as the QGS technique has a high reproducibility (for each individual center) ${ }^{7,8,35}$ the data on $\mathrm{LV}$ function can be used for risk stratification.

It would have been interesting to demonstrate whether LV volumes add prognostic information over LVEF. However, both variables are strongly interrelated, implying that LV volumes and LVEF cannot simultaneously be incorporated in a statistical model. Consequently, based on our data it is virtually impossible to decide whether LV volumes add incremental value to LVEF.

In the present study, we included all female patients who underwent a myocardial perfusion study, without screening patients for pre-test likelihood of CAD. However, according to the used ESC guidelines, most patients have an intermediate likelihood of CAD. In the present study, ischemia did not show independent incremental prognostic value for predicting any cardiac events, but the study population was relatively small, the follow-up period was rather short and not for $100 \%$ complete (a total of $<5 \%$ lost to follow up), and the 
number of events was low. Future studies will be needed to further separate the group of patients with combined perfusion and functional high risk features.

\section{CONCLUSION}

In women, perfusion and functional parameters derived from quantitative gated technetium-99m tetrofosmin SPECT imaging can adequately be used for cardiac risk assessment. Using quantitative gated SPECT, female patients with an LVEF $<52 \%$ or an SSS $\geq 22$ are at increased risk for subsequent hard events. Women with an SSS $\geq 14$ are at increased risk for any cardiac events.

\section{Open Access}

This article is distributed under the terms of the Creative Commons Attribution Noncommercial License which permits any noncommercial use, distribution, and reproduction in any medium, provided the original author(s) and source are credited.

\section{References}

1. American Heart Association. Heart and stroke facts: 2002 statistical supplement. Dallas: American Heart Association; 2002.

2. Mosca L, Grundy S, Judelson D, King K, Limacher M, Oparil S, et al. AHA scientific statement: Consensus panel statement. Guide to preventive cardiology in women. Circulation 1999;99:2480-4.

3. Vaccarino V, Parsons L, Every NR, Barron HV, Krumholz HM. Sex-based differences in early mortality after myocardial infarction. National Registry of Myocardial infarction 2 participants. N Engl J Med 1999;341:217-25.

4. Mieres JM, Shaw LJ, Arai A, Budoff MJ, Flamm SD, Hundley WG, et al. Role of non-invasive testing in the clinical evaluation of women with suspected coronary artery disease. AHA scientific statement. Circulation 2005;111:682-96.

5. Mieres JH, Shaw LJ, Hendel RC, Miller DD, Bonow RO, Berman DS, et al. A report of the American Society of Nuclear Cardiology task force on women and heart disease (writing group on perfusion imaging in women). J Nucl Cardiol 2003;10:95-101.

6. Kasai T, DePuey EG, Shah AA. Decreased septal wall thickening in patients with left bundle branch block. J Nucl Cardiol 2004; 11:32-7.

7. Germano G, Kavanagh PB, Waechter P, Areeda J, Van Kriekinge $\mathrm{S}$, Sharir $\mathrm{T}$, et al. A new algorithm for the quantitation of myocardial perfusion SPECT. 1: technical principles and reproducibility. J Nucl Med 2000;41:712-9.

8. Sharir T, Germano G, Waechter PB, Kavanagh PB, Areeda JS, Gerlach $\mathrm{J}$, et al. A new algorithm for the quantitation of myocardial perfusion SPECT. 2: Validation and diagnostic yield. J Nucl Med 2000;41:720-7.

9. Sharir T, Germano G, Kavanagh PB, Lai S, Cohen I, Lewin HC, et al. Incremental prognostic value of post-stress LV ejection fraction and volume by gated myocardial perfusion single photon emission computed tomography. Circulation 1999;100:1035-42.

10. Travin MI, Heller GV, Johnson LL, Katten D, Ahlberg AW, Isasi $\mathrm{CR}$, et al. The prognostic value of ECG-gated SPECT imaging in patients undergoing stress Tc-99m sestamibi myocardial perfusion imaging. J Nucl Cardiol 2004;11:253-62.

11. Sharir T, Kang X, Germano G, Bax J, Shaw L, Gransar H, et al. Prognostic value of poststress left ventricular volume and ejection fraction by gated myocardial perfusion SPECT in women and men: gender-related differences in normal limits and outcomes. J Nucl Cardiol 2006;13:495-506.

12. Nakajima K, Nishimura S, Nishimura T. Normal limits of ejection fraction and volumes determined by gated SPECT in clinically normal patients without cardiac events: a study on the J-ACCESS database. Eur J Nucl Med Mol Imaging 2007;34:1088-96.

13. Verberne H, Dibbets-Schneider P, Spijkerboer A, Stokkel M, van Eck-Smit B, Busemann Sokole E. Multicenter intercomparison assessment of consistency of left ventricular function from gated cardiac SPECT phantom. J Nucl Cardiol 2006;13:801-10.

14. Berman D, Abidov A, Kang X, Hayes S, Friedman J, Sciammarella M, et al. Prognostic validation of a 17-segment score derived from a 20-segment score for myocardial perfusion SPECT interpretation. J Nucl Cardiol 2004;11:414-23.

15. Van Eck-Smit BL, Poots S, Zwinderman AH, Bruschke AV, Pauwels EK, Van der Wall EE. Myocardial SPET imaging with 99mTc-tetrofosmin in clinical practice: comparison of a 1 day and a 2 day imaging protocol. Nucl Med Commun 1997;18: 24-30.

16. Berman DS, Kang X, van Train KF, Lewin HC, Cohen I, Areeda J, et al. Comparative prognostic value of automatic quantitative analysis versus semiquantitative visual analysis of exercise myocardial perfusion single-photon emission computed tomography. J Am Coll Cardioll 1998;32:1987-95.

17. Hachamovitch R, Hayes SW, Friedman JD, Cohen I, Berman DS. A prognostic score for prediction of cardiac mortality risk after adenosine stress myocardial perfusion scintigraphy. J Am Coll Cardiol 2005;45:722-9.

18. Berman DS, Wong ND, Gransar H, Miranda-Peats R, Dahlbeck J, Hayes SW, et al. Relationship between stress-induced myocardial ischemia and atherosclerosis measured by coronary calcium tomography. J Am Coll Cardiol 2004;44:923-30.

19. Concato J, Peduzzi P, Holfold TR, Feinstein AR. Importance of events per independent variable in proportional hazards analysis. I. Background, goals, and general strategy. J Clin Epidemiol 1995; 48:1495-501.

20. Peduzzi P, Concato J, Kemper E, Holford TR, Feinstein AR. A simulation study of the number of events per variable in logistic regression analysis. J Clin Epidemiol 1996;49:1373-9.

21. Vittinghoff E, McCulloch CE. Relaxing the rule of ten events per variable in logistic and Cox regression. Am J Epidemiol 2007; 165(6):710-8.

22. Galassi AR, Azzarelli S, Tomaselli A, Giosofatto R, Ragusa A, Musumeci S, et al. Incremental prognostic value of technetium$99 \mathrm{~m}$-tetrofosmin exercise myocardial perfusion imaging for predicting outcomes in patients with suspected or known coronary artery disease. Am J Cardiol 2001;88:101-6.

23. Schinkel A, Elhendy A, van Domburg R, Bax J, Vourvouri E, Bountioukos M, et al. Incremental value of exercise technetium$99 \mathrm{~m}$ tetrofosmin myocardial perfusion single-photon emission computed tomography for the prediction of cardiac events. Am J Cardiol 2003;91:408-11.

24. Groutars R, Verzijlbergen F, Zwinderman A, Tiel-van Buul M, Ascoop C, van Hemel N, et al. Incremental prognostic value of myocardial SPET with dual-isotope rest ${ }^{201} \mathrm{Tl} / \mathrm{stress}{ }^{99 \mathrm{~m}} \mathrm{Tc}$-tetrofosmin. Eur J Nucl Med 2002;29:46-52.

25. Petix NR, Sestini S, Coppola A, Marcucci G, Nassi F, Taiti A, et al. Prognostic value of combined perfusion and function by stress technetium-99m sestamibi gated SPECT myocardial 
perfusion imaging in patients with suspected or known coronary artery disease. Am J Cardiol 2005;95(11):1351-7.

26. Sharir T, Germano G, Kang X, Lewin HC, Miranda R, Cohen I, et al. Prediction of myocardial infarction versus cardiac death by gated myocardial perfusion SPECT: risk stratification by the amount of stress-induced ischemia and poststress ejection fraction. J Nucl Med 2001;42:831-7.

27. De Winter O, van de Veire N, De Bondt P, Van Der Wiele C, Buyzere M, De Backer G, et al. Poststress left ventricular ejection fraction is an independent predictor of major cardiac events in patients with coronary artery disease and impaired left ventricular function. Q J Nucl Med Mol Imaging 2008;52:296-304.

28. Zellweger MJ, Dubois EA, Lai S, Shaw LJ, Amanullah AM, Lewin $\mathrm{HC}$, et al. Risk stratification in patients with remote prior myocardial infarction using rest-stress myocardial perfusion SPECT: prognostic value and impact on referral to early catheterization. J Nucl Cardiol 2002;9:23-32.

29. Groutars RG, Verzijlbergen JF, Muller A, Ascoop C, Tiel-van Buul MM, Zwinderman AH, et al. Prognostic value and quality of life in patients with normal rest thallium-201/stress technetium 99m-tetrofosmin dual-isotope myocardial SPECT. J Nucl Cardiol 2000;7:333-41.

30. Schinkel AF, Elhendy A, van Domburg RT, Bax JJ, Vourvouri EC, Bountioukos M, et al. Incremental value of exercise technetium-
$99 \mathrm{~m}$ tetrofosmin myocardial perfusion single-photon emission computed tomography for the prediction of cardiac events. Am J Cardiol 2003;91:408-11.

31. Sharir T. The role of regional myocardial dysfunction by gated myocardial perfusion SPECT in the prognostic evaluation of patients with coronary artery disease. J Nucl Cardiol 2005;12: 20-31.

32. Nallamothu N, Bagheri B, Acio ER, Heo J, Iskandrian AE. Prognostic value of stress myocardial perfusion single photon emission computed tomography imaging in patients with left bundle branch block. J Nucl Cardiol 1997;4:550-2.

33. Gil VM, Almeida M, Ventosa A. Prognosis in patients with left bundle branch block and normal dipyridamole thallium-201 scintigraphy. J Nucl Cardiol 1998;5:414-7.

34. Nigam A, Humen DP. Prognostic value of myocardial perfusion imaging with exercise and/or dipyridamole hyperemia in patients with preexisting left bundle branch block. J Nucl Med 1998;39: $579-81$.

35. America YG, Bax JJ, Dibbets-Schneider P, Pauwels EK, Van der Wall EE. Evaluation of the Quantitative Gated SPECT (QGS) software program in the presence of large perfusion defects. Int $\mathbf{J}$ Cardiovasc Imaging 2005;21:519-29. 